\title{
Attempts and approximations for a background modeling in pharmaceutical samples in patterns of XRD and S-XRPD
}

\author{
Bárbara A. Ramírez Almaguer ${ }^{* 1}$, Lauro Bucio ${ }^{1}$, Mathilde Reinle-Schmitt ${ }^{2}$, Antonio Cervellino ${ }^{3}$ \\ 1 Instituto de Física, Universidad Nacional Autónoma de México, Circuito de la Investigación \\ Científica s/n Ciudad Universitaria, Coyoacán, México, D.F., 04150, México, D.F. \\ 2 Excelsus Structura Solutions, Villigen, Switzerland. \\ ${ }^{3}$ Paul Scherrer Institute, Villigen Switzerland. \\ email: bara219@gmail.com
}

Pharmaceutical solids can exist in several forms: polymorphic crystalline and amorphous arrangements. They present differences in their physicochemical properties, for instance, melting point, density, morphology, solubility, and color. These characteristics may have an impact on the stability (physical and chemical), bioavailability and bioequivalencel; e.g., amorphous substances are unstable than crystalline substances. Differences in degrees of drug crystallization affect chemical and physical stability, rather than crystalline polymorphism of the substance. Tablets are solids preparations of active pharmaceutical ingredients and additives; many of the additives commonly used are poorly crystalline or amorphous, determining the final solubility in the drug.

A proper background modeling is the first step for correcting amorphous fraction quantification in active pharmaceutical ingredients, additives, and mixes.

A python-code based program to model the background of the diffraction patterns as a contribution of diffuse scattering -Thermal Diffuse Scattering (TDS) plus static disorder, Compton and air scattering-, according to the present crystalline phases; Air scattering based in correction factors for absorption and air scattering under a symmetrical reflection geometry with given sample thickness, divergence and receiving slit width, average temperature factors and specimen density of packing has been developed and probe in blends of ciprofloxacin (API-Cipro) and microcrystalline cellulose (MC) in different blends had been performed using the program.

As a second approximation to this subject, a series of S-XRPD diffraction patterns from three highly crystalline reference samples had been collected at the MS beamline (PD End station) in the Swiss Light Source to understand the contribution of the capillary, air and sample absorption besides the packing density in the contributions to the diffuse background. 